\title{
KIT I NACHFOLGE FÜR PROFESSOR SPICHER
}

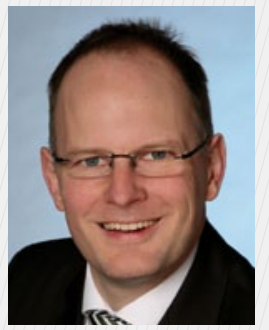

Am Institut für Kolbenmaschinen (IFKM) des Karlsruher Instituts für Technologie (KIT) forschen die Wissenschaftler unter neuer Leitung. Prof. Dr. sc. techn. THOMAS KOCH hat die Nachfolge von Prof. Dr.-Ing. Ulrich Spicher angetreten. Koch war zuvor zehn Jahre lang bei Daimler tätig. Er wirkte bei der Entwicklung der neuen Medium-Duty-Dieselmotorenbaureihe 0M934/936 mit und begleitete diese von der Konzeptfestlegung bis zum Serienstart im Jahr
2013. Als Leiter Grundlagen/Verbrennung verantwortete er bei Daimler unter anderem die Auslegung von Brennverfahren, Ansaugluft-Management oder Kraftstoffen. Wichtige Forschungsschwerpunkte am IKFM sollen künftig vor allem die Gesamtsystementwicklung unter Einbeziehung der Abgasnachbehandlung und Restwärmenutzung sein. Die aktuellen Aktivitäten im Bereich der Brennverfahrensanalyse für verschiedene Kraftstoffe sollen ebenfalls weitergeführt werden.

\section{AFT I JETZT SCHAEFFLER ENGINEERING}

Die AFT Atlas Fahrzeugtechnik GmbH firmiert seit dem 1. März 2013 unter SCHAEFFLER ENGINEERING. Die Umbenennung des Entwicklungsdienstleisters ist Teil der neuen Unternehmensstrategie, mit der sich das mittelständische Unternehmen weltweit als Engineering-Dienstleister und Systemspezialist für Mechatronik positionieren will. AFT mit Sitz in Werdohl wurde 1979 gegründet, seit 2004 ist das Unternehmen zu $100 \%$ im Besitz von Schaeffler (Zentrale in Herzogenaurach, Bild). Schaeffler Engineering bietet Dienstleistungen und Produkte vor allem in den Bereichen Mechatronik und Systementwicklung, aber auch bei Getriebeentwicklung, Fahrzeugakustik, Prototypenbau sowie bei der Kleinserienfertigung elektronischer Steuergeräte oder Messsysteme an.

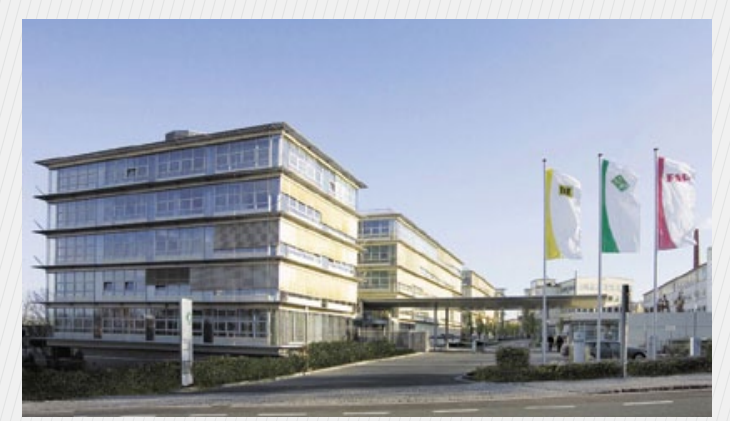

\section{POLYTEC I WECHSEL IN GESCHÄFTSFÜHRUNG}

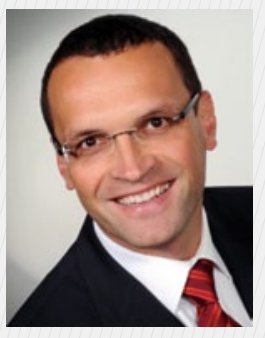

ALFRED LINK ist neuer Geschäftsführer für den kaufmännischen Bereich bei Polytec. Er teilt sich die Geschäftsführung mit Dr. Hans Lothar Pasch, der bereits seit 2009 als Geschäftsführer für die Bereiche Vertrieb und Technik verantwortlich ist. Der Wirtschaftsingenieur Link war nach dem Studium an der Fachhochschule Heilbronn erst bei Scania in Koblenz und dann bei Behr in Stuttgart tätig. Im Jahr 2003 wech- selte er zu Groz-Beckert, wo er unter anderem vier Jahre kaufmännischer Leiter der Tochtergesellschaft Indien war, bevor er 2011 zu Polytec wechselte. Bevor Link die Geschäftsführung übernahm, war er kaufmännischer Leiter bei Polytec. Sein Vorgänger Dr. Karl Spanner, der nach 21 Jahren seinen Posten an Link überantwortete, werde das Unternehmen weiterhin als Gesellschafter unterstützen.

\section{BMW MOTORRAD I TRENNUNG VON HUSQVARNA}

Der Geschäftsbereich Motorrad der BMW Group wird strategisch neu aufgestellt und fokussiert sich nun auf die Schwerpunkte urbane Mobilität und E-Mobilität. BMW verkauft daher die italienische Motocross-Motorradmarke Husqvarna, in Schweden 1904 gegründet, an das österreichische Unternehmen PIERER INDUSTRIE, zu dem auch KTM gehört. Mit der Konzentration auf den Ausbau der Marke BMW Motorrad im Bereich urbane Mobilität und E-Mobilität reagiert der Hersteller auf aktuelle demografische Entwicklungen und wachsende Umweltanforderungen. Husqvarna ist seit 2007 ein unabhängiges Tochterunternehmen der BMW Group gewesen. Der Hauptsitz liegt seit 2009 in Biandronno nahe Varese (Italien).

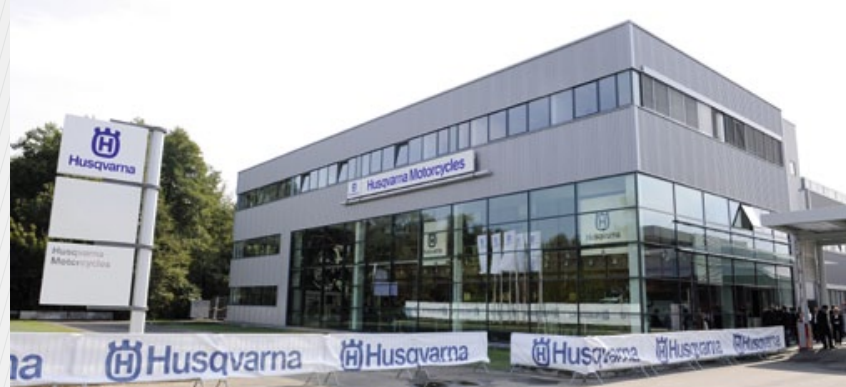

